\title{
Article
}

\section{On characteristic polynomial and energy of Sombor matrix}

\author{
Gowtham Kalkere Jayanna ${ }^{1}$ and Ivan Gutman ${ }^{2, *}$ \\ 1 Department of Mathematics, University College of Science, Tumkur University, Tumakuru, India. \\ 2 Faculty of Science, University of Kragujevac, 34000 Kragujevac, Serbia. \\ * Correspondence: gutman@kg.ac.rs
}

Academic Editor: Aisha Javed

Received: 1 October 2021; Accepted: 25 October 2021; Published: 31 October 2021.

Abstract: Let $G$ be a simple graph with vertex set $V=\left\{v_{1}, v_{2}, \ldots, v_{n}\right\}$, and let $d_{i}$ be the degree of the vertex $v_{i}$. The Sombor matrix of $G$ is the square matrix $\mathbf{A}_{S O}$ of order $n$, whose $(i, j)$-element is $\sqrt{d_{i}^{2}+d_{j}^{2}}$ if $v_{i}$ and $v_{j}$ are adjacent, and zero otherwise. We study the characteristic polynomial, spectrum, and energy of $\mathbf{A}_{S O}$. A few results for the coefficients of the characteristic polynomial, and bounds for the energy of $\mathbf{A}_{S O}$ are established.

Keywords: Sombor index; Sombor matrix; Energy (of Sombor matrix); Characteristic polynomial (of Sombor matrix); Degree (of vertex).

MSC: 05C07; 05C09; 05C92.

\section{Introduction}

$\mathbf{T}$ he Sombor index $S O$ is a recently introduced vertex-degree-based topological index [1]. It promptly attracted much attention and its mathematical properties and chemical applications became a topic of a remarkably large number of studies, e.g., [2-9]. Also promptly, the concept of Sombor index was extended to linear algebra, by defining the Sombor matrix, which then led to the investigation of its spectrum and various spectrum-based properties [10-14]. In particular, the energy of the Sombor matrix was much examined [11-14]. In the present paper we report a few additional results on this matter, with emphasis on the characteristic polynomial and energy.

In this paper, we considered simple, finite, undirected, and connected graphs. Let $G$ be such a graph, with vertex set $\mathbf{V}(G)$ and edge set $\mathbf{E}(G)$. If two vertices have a common edge then they are said to be adjacent. If the vertices $u$ and $v$ are adjacent, then the edge connecting them is denoted by $u v$. The number of edges incident to a vertex $v$ is called the degree of that vertex $v$, and is denoted by $d_{v}$.

In the mathematical and chemical literature, a great number of vertex-degree-based graph invariants of the form

$$
T I=T I(G)=\sum_{u v \in \mathbf{E}(G)} \varphi\left(d_{u}, d_{v}\right)
$$

have been considered, where $\varphi$ is a suitably chosen function, with property $\varphi(x, y)=\varphi(y, x)$. These invariants are usually referred to as topological indices. Among them are the forgotten topological index [15]

$$
F(G)=\sum_{u v \in \mathbf{E}(G)}\left(d_{u}^{2}+d_{v}^{2}\right)=\sum_{u \in \mathbf{V}(G)} d_{u}^{3}
$$

the Sombor index [1]

$$
S O(G)=\sum_{u v \in \mathbf{E}(G)} \sqrt{d_{u}^{2}+d_{v}^{2}}
$$

and many other [16,17]. 
The adjacency matrix $\mathbf{A}(G)=\left(a_{i j}\right)_{n \times n}$ of the graph $G$ with vertex set $\mathbf{V}(G)=\left\{v_{1}, v_{2}, \ldots, v_{n}\right\}$, is the symmetric matrix of order $n$, whose elements are defined as [18]:

$$
a_{i j}= \begin{cases}1 & \text { if } v_{i} v_{j} \in \mathbf{E}(G) \\ 0 & \text { if } v_{i} v_{j} \notin \mathbf{E}(G) \\ 0 & \text { if } i=j .\end{cases}
$$

The characteristic polynomial of $\mathbf{A}(G)$ is $\phi(G, \lambda)=\operatorname{det}\left[\lambda \mathbf{I}_{n}-\mathbf{A}(G)\right]$, where $\mathbf{I}_{n}$ is the unit matrix of order $n$ [18]. The eigenvalues $\lambda_{1}, \lambda_{2}, \ldots, \lambda_{n}$ of $\mathbf{A}(G)$ form the spectrum of the graph $G$ [18]. Recall that these eigenvalues coincide with the zeros of $\phi(G, \lambda)$.

The energy of the graph $G$ is defined as [19]:

$$
\operatorname{En}(G)=\sum_{i=1}^{n}\left|\lambda_{i}\right|
$$

The theory of graph spectra, including the theory of graph energy, is nowadays a well elaborated part of discrete mathematics. In parallel with the above specified graph-spectral concepts, we now introduce their Sombor-index-related counterparts. The following definition is an application to the Sombor index of the general spectral theory of matrices associated with vertex-degree-based topological indices of the form (1) [20-22].

Definition 1. (1) The Sombor matrix $\mathbf{A}_{S O}(G)=\left(s o_{i j}\right)_{n \times n}$ of the graph $G$ with vertex set $\mathbf{V}(G)=$ $\left\{v_{1}, v_{2}, \ldots, v_{n}\right\}$, is the symmetric matrix of order $n$, whose elements are

$$
s o_{i j}=\left\{\begin{array}{cl}
\sqrt{d_{v_{i}}^{2}+d_{v_{j}}^{2}} & \text { if } v_{i} v_{j} \in \mathbf{E}(G) \\
0 & \text { if } v_{i} v_{j} \notin \mathbf{E}(G) \\
0 & \text { if } i=j .
\end{array}\right.
$$

(2) The Sombor characteristic polynomial of the graph $G$ is $\phi_{S O}(G, \lambda)=\operatorname{det}\left[\lambda \mathbf{I}_{n}-\mathbf{A}_{S O}(G)\right]$. We will write it in the form

$$
\phi_{S O}(G, \lambda)=\sum_{k \geq 0} s o(G, k) \lambda^{n-k} .
$$

(3) The eigenvalues $\sigma_{1}, \sigma_{2}, \ldots, \sigma_{n}$ of the Sombor matrix $\mathbf{A}_{S O}(G)$ form the Sombor spectrum of the graph $G$.

(4) The Sombor energy of the graph $G$ is

$$
\operatorname{En}_{S O}(G)=\sum_{i=1}^{n}\left|\sigma_{i}\right|
$$

Since $\mathbf{A}_{S O}(G)$ is a real symmetric matrix, all its eigenvalues, i.e., all roots of $\phi_{S O}(G, \lambda)=0$, are real. Thus, they can be arranged as $\sigma_{1} \geq \sigma_{2} \geq \cdots \geq \sigma_{n}$.

Remark 1. Comparing Equations (2) and (3), we see that the Sombor matrix can be viewed as the ordinary adjacency matrix of a graph with weighted edges, such that the weight of the edge $v_{i} v_{j}$ is $\sqrt{d_{v_{i}}^{2}+d_{v_{j}}^{2}}$. This observation allows us to apply to the Sombor matrix and its spectrum the standard methods of graph spectral theory [18], in particular the Sachs coefficient theorem [23].

\section{Preliminaries}

The following elementary spectral properties of the Sombor matrix were recognized in several earlier studies [10-14].

Lemma 1. Let $G$ be a graph with Sombor eigenvalues $\sigma_{1}, \sigma_{2}, \ldots, \sigma_{n}$. Then

$$
\sum_{i=1}^{n} \sigma_{i}=0
$$




$$
\begin{aligned}
& \sum_{i=1}^{n} \sigma_{i}^{2}=2 F(G) \\
& \sum_{i=1}^{n} \sigma_{i}^{3}=6 \sum_{\Delta} \prod_{u v \in \mathbf{E}(\Delta)} \sqrt{d_{u}^{2}+d_{v}^{2}}
\end{aligned}
$$

or, equivalently,

$$
\begin{aligned}
& \operatorname{so}(G, 1)=0, \\
& \operatorname{so}(G, 2)=-F(G), \\
& \operatorname{so}(G, 3)=-2 \sum_{\Delta} \prod_{u v \in \mathbf{E}(\Delta)} \sqrt{d_{u}^{2}+d_{v}^{2}},
\end{aligned}
$$

where $\sum_{\Delta}$ indicates summation over all triangles contained in the graph $G$.

Formula (6) can be generalized as follows:

Lemma 2. Let $p$ be the size of smallest odd cycle contained in the graph $G$, and let $\sum_{C_{p}}$ indicate summation over all cycles of size $p$ contained in $G$. Then for $q=1,3, \ldots, p-2$,

$$
\sum_{i=1}^{n} \sigma_{i}^{q}=0
$$

whereas

$$
\sum_{i=1}^{n} \sigma_{i}^{p}=2 p \sum_{C_{p}} \prod_{u v \in \mathbf{E}\left(C_{p}\right)} \sqrt{d_{u}^{2}+d_{v}^{2}}
$$

or, equivalently,

$$
s o(G, p)=-2 \sum_{C_{p}} \prod_{u v \in \mathbf{E}\left(C_{p}\right)} \sqrt{d_{u}^{2}+d_{v}^{2}} .
$$

If $G$ does not possess odd cycles, i.e., if $G$ is bipartite, then relations (7) and $\operatorname{so}(G, q)=0$ hold for all odd values of $q$.

Proof. Take into account Remark 1, and use the analogous result for ordinary graphs [18].

Lemma 3. [24,25] Suppose that $a_{i}$ and $b_{i}$ are non negative real numbers for $1 \leq i \leq n$. Then,

$$
\left(\sum_{i=1}^{n} a_{i}^{2}\right)\left(\sum_{i=1}^{n} b_{i}^{2}\right) \leq \frac{1}{4}\left(\sqrt{\frac{M_{1} M_{2}}{m_{1} m_{2}}}+\sqrt{\frac{m_{1} m_{2}}{M_{1} M_{2}}}\right)^{2}\left(\sum_{i=1}^{n} a_{i} b_{i}\right)^{2}
$$

where $M_{1}=\max _{1 \leq i \leq n} a_{i}, M_{2}=\max _{1 \leq i \leq n} b_{i}, m_{1}=\min _{1 \leq i \leq n} a_{i}$, and $m_{2}=\min _{1 \leq i \leq n} b_{i}$

Lemma 4. [24,25] Using the same notation as in Lemma 3,

$$
\left(\sum_{i=1}^{n} a_{i}^{2}\right)\left(\sum_{i=1}^{n} b_{i}^{2}\right)-\left(\sum_{i=1}^{n} a_{i} b_{i}\right)^{2} \leq \frac{n^{2}}{4}\left(M_{1} M_{2}-m_{1} m_{2}\right)^{2} .
$$

\section{New bounds for Sombor energy}

Various lower and upper bounds for Sombor energy were already reported in [10-13]. In this section we establish a few more.

We first recall a result by Lin and Miao [13], that can be stated in terms of traces of the Sombor matrix. It should be compared with the below Theorem 2. The upper bound was obtained also in [12]. Note that $\operatorname{tr}\left(\mathbf{A}_{S O}(G)^{2}\right)=2 F(G)$ follows from Equation (5). 
Theorem 1. [13] Denote the trace of a square matrix $\mathbf{M}$ by $\operatorname{tr}(\mathbf{M})$. Let $G$ be a graph on $n$ vertices. Then

$$
\sqrt{\operatorname{tr}\left(\mathbf{A}_{S O}(G)^{2}\right)} \leq E n_{S O}(G) \leq \sqrt{n \operatorname{tr}\left(\mathbf{A}_{S O}(G)^{2}\right)}
$$

i.e.,

$$
\sqrt{2 F(G)} \leq E n_{S O}(G) \leq \sqrt{2 n F(G)} .
$$

Theorem 2. Let $G$ be a non-trivial graph. Then

$$
E n_{S O}(G) \geq \sqrt{\frac{\left[\operatorname{tr}\left(\mathbf{A}_{S O}(G)^{2}\right)\right]^{3}}{\operatorname{tr}\left(\mathbf{A}_{S O}(G)^{4}\right)}} .
$$

Proof. By the Hölder inequality,

$$
\sum_{i=1}^{n} a_{i} b_{i} \leq\left(\sum_{i=1}^{n} a_{i}^{p}\right)^{1 / p}\left(\sum_{i=1}^{n} b_{i}^{q}\right)^{1 / q}
$$

where, $a_{i}, b_{i} \in \mathbf{R}^{+},(i=1,2,3 \ldots, n)$. Setting $a_{i}=\left|\sigma_{i}\right|^{2 / 3}, b_{i}=\left|\sigma_{i}\right|^{4 / 3}, p=3 / 2$, and $q=3$, we get

$$
\sum_{i=1}^{n}\left|\sigma_{i}\right|^{2} \leq\left(\sum_{i=1}^{n}\left|\sigma_{i}\right|\right)^{2 / 3}\left(\sum_{i=1}^{n}\left|\sigma_{i}\right|^{4}\right)^{1 / 3}
$$

which by Equation (4), and bearing in mind that since $G$ is not an empty graph and thus $\sum_{i=1}^{n}\left|\sigma_{i}\right|^{4} \neq 0$, yields Theorem 2.

Theorem 3. If $\sigma_{1}$ is the greatest Sombor eigenvalue, then $E n_{S O}(G) \leq 2 \sigma_{1}$. For connected graphs, equality holds if and only if $G$ is a complete bipartite graph.

Proof. Bearing in mind Equation (4),

$$
\operatorname{En}_{S O}(G)=\left|\sigma_{1}\right|+\sum_{i=2}^{n}\left|\sigma_{i}\right| \geq\left|\sigma_{1}\right|+\left|\sum_{i=2}^{n} \sigma_{i}\right| .
$$

On the other hand,

$$
\sum_{i=1}^{n} \sigma_{i}=0 \quad \Longrightarrow \quad \sigma_{1}=-\sum_{i=2}^{n} \sigma_{i} \text { and so } \quad\left|\sigma_{1}\right|=\left|\sum_{i=2}^{n} \sigma_{i}\right| .
$$

Equality holds if $\sigma_{1}$ and $\sigma_{n}$ are the only non-zero eigenvalues. In view of Remark 1, this happens only if $G$ is a complete bipartite graph.

Theorem 4. Let $G$ be a graph with $n$ vertices. If no Sombor eigenvalue of $G$ is equal to zero, then

$$
E n_{S O}(G) \geq \sqrt{\frac{8 n F(G) \sigma_{\ell} \sigma_{s}}{\left|\sigma_{\ell}\right|+\left|\sigma_{s}\right|}}
$$

where, $\left|\sigma_{\ell}\right|$ and $\left|\sigma_{s}\right|$ are, respectively, the largest and smallest absolute values of the eigenvalues in the Sombor spectrum of $G$. Of course, $\left|\sigma_{\ell}\right|=\sigma_{1}$.

Proof. Setting in Lemma 3, $a_{i}=\left|\sigma_{i}\right|$ and $b_{i}=1$ for $1 \leq i \leq n$, we get

$$
\left(\sum_{i=1}^{n}\left|\sigma_{i}\right|^{2}\right)\left(\sum_{i=1}^{n} 1\right) \leq \frac{1}{4}\left(\sqrt{\frac{\left|\sigma_{\ell}\right|}{\left|\sigma_{s}\right|}}+\sqrt{\frac{\left|\sigma_{s}\right|}{\left|\sigma_{\ell}\right|}}\right)^{2}\left(\sum_{i=1}^{n}\left|\sigma_{i}\right|\right)^{2},
$$


where $\left|\sigma_{\ell}\right|=\max _{1 \leq i \leq n}\left\{\left|\sigma_{i}\right|\right\}$ and $\left|\sigma_{s}\right|=\min _{1 \leq i \leq n}\left\{\left|\sigma_{i}\right|\right\}$. Then

$$
2 F(G) n \leq \frac{1}{4}\left(\sqrt{\frac{\left|\sigma_{\ell}\right|}{\left|\sigma_{s}\right|}}+\sqrt{\frac{\left|\sigma_{s}\right|}{\left|\sigma_{\ell}\right|}}\right)^{2}\left(\sum_{i=1}^{n}\left|\sigma_{i}\right|\right)^{2}
$$

and thus

$$
\sqrt{8 n F(G)} \leq\left(\frac{\left|\sigma_{\ell}\right|+\left|\sigma_{S}\right|}{\sqrt{\sigma_{\ell} \sigma_{s}}}\right) E n_{S O}(G)
$$

which straightforwardly leads to Theorem 4.

Theorem 5. Let $G$ be a connected graph with $n$ vertices, and $\sigma_{\ell}, \sigma_{s}$ same as in Theorem 4 . Then

$$
E n_{S O}(G) \geq \sqrt{2 n F(G)-\frac{n^{2}}{4}\left(\left|\sigma_{\ell}\right|-\left|\sigma_{S}\right|\right)}
$$

Proof. Setting in Lemma $4, a_{i}=\left|\sigma_{i}\right|$ and $b_{i}=1$ for $1 \leq i \leq n$, we get

$$
\left(\sum_{i=1}^{n}\left|\sigma_{i}\right|^{2}\right)\left(\sum_{i=1}^{n} 1\right)-\left(\sum_{i=1}^{n}\left|\sigma_{i}\right|\right)^{2} \leq \frac{n^{2}}{4}\left(\left|\sigma_{\ell}\right|-\left|\sigma_{s}\right|\right)^{2},
$$

implying

$$
2 F(G) n-E n_{S O}(G)^{2} \leq \frac{n^{2}}{4}\left(\left|\sigma_{\ell}\right|-\left|\sigma_{s}\right|\right)^{2} .
$$

Theorem 5 follows.

\section{On Sombor energy of trees}

In this section we focus our attention to trees. Let $T$ be a tree on $n$ vertices, $n \geq 2$. The main result in the spectral theory of trees is the formula $[18,26,27]$

$$
\phi(T, \lambda)=\lambda^{n}+\sum_{k \geq 1}(-1)^{k} m(T, k) \lambda^{n-2 k}
$$

where $m(T, k)$ stands for the number of $k$-matchings (= selections of $k$ mutually independent edges) in the tree $T$. By definition, $m(T, 1)=n-1$.

As explained in Remark 1, the matrix $\mathbf{A}_{S O}(G)$ can be viewed as the adjacency matrix of a graph with weighted edges. This, of course, applies also to trees.

According to the Sachs coefficient theorem [18,23], for the Sombor characteristic polynomial of a tree $T$, an expression analogous to Equation (8) would hold, namely

$$
\phi_{S O}(T, \lambda)=\lambda^{n}+\sum_{k \geq 1}(-1)^{k} m_{S O}(T, k) \lambda^{n-2 k} .
$$

The coefficient $m_{S O}(T, k)$ is equal to the sum of weights coming from all $k$-matchings of $T$. Each particular $k$-matching contributes to $m_{S O}(T, k)$ by the product of the squares of the terms $\sqrt{d_{u}^{2}+d_{v}^{2}}$, pertaining to the edges contained in that matching [23]. Thus, let $M$ be a distinct $k$-matching of $T$, and let $\mathcal{M}(k)$ be the set of all such $k$-matchings. Then for $k \geq 1, \mathcal{M}(k)$ consists of $m(T, k)$ elements, i.e., $|\mathcal{M}(k)|=m(T, k)$.

The weight of a single matching $M$ is equal to $\prod_{u v \in M}\left(d_{u}^{2}+d_{v}^{2}\right)$ and therefore

$$
m_{S O}(T, k)=\sum_{M \in \mathcal{M}(k)} \prod_{u v \in M}\left(d_{u}^{2}+d_{v}^{2}\right)
$$

provided $\mathcal{M}(k) \neq \varnothing$. If, on the other hand, $\mathcal{M}(k)=\varnothing$, then $m_{S O}(T, k)=0$.

We thus see that the coefficients $m_{S O}(T, k)$ are positive if $m(T, k)>0$ and are equal to zero if $m(T, k)=0$. This implies: 
Theorem 6. The inertia of the Sombor matrix and of the ordinary adjacency matrix of any tree coincide.

The energy of a tree can be computed from its matching polynomial as [28]:

$$
E n(T)=\frac{2}{\pi} \int_{0}^{\infty} \frac{d x}{x^{2}} \ln \left[1+\sum_{k \geq 1} m(T, k) x^{2 k}\right] d x .
$$

The analogous expression for the Sombor energy is

$$
E n_{S O}(T)=\frac{2}{\pi} \int_{0}^{\infty} \frac{d x}{x^{2}} \ln \left[1+\sum_{k \geq 1} m_{S O}(T, k) x^{2 k}\right] d x
$$

and can be obtained in the exactly same manner as Equation (11) $[28,29]$.

Since, evidently, $m_{S O}(T, k)>m(T, k)$ holds whenever the tree $T$ has at least one $k$-matching, by comparing Equations (11) and (12), we immediately arrive at:

Theorem 7. For any tree $T, E n_{S O}(T)>E n(T)$.

Author Contributions: All authors contributed equally to the writing of this paper. All authors read and approved the final manuscript.

Conflicts of Interest: "The authors declare no conflict of interest."

\section{Bibliography}

[1] Gutman, I. (2021). Geometric approach to degree-based topological indices: Sombor indices. MATCH Communications in Mathematical and in Compututer Chemistry, 86, 11-16.

[2] Aguilar-Sánchez, R., Méndez-Bermúdez, J. A., Rodríguez, J. M., \& Sigarreta, J. M. (2021). Normalized Sombor indices as complexity measures of random graphs. Entropy, 23(8), Article No. 976, https://doi.org/10.3390/e23080976.

[3] Cruz, R., Rada, J., \& Sigarreta, J. M. (2021). Sombor index of trees with at most three branch vertices. Applied Mathematics and Computation, 409, Article No. 126414, https://doi.org/10.1016/j.amc.2021.126414.

[4] Das, K. C., \& Gutman, I. (2022). On Sombor index of trees. Applied Mathematics and Computation, 412, Article No. 126525, https://doi.org/10.1016/j.amc.2021.126575.

[5] Das, K. C., \& Shang, Y. (2021). Some extremal graphs with respect to Sombor index. Mathematics, 9(11), Article No. 1202, https://doi.org/10.3390/math9111202.

[6] Gutman, I. (2021). Some basic properties of Sombor indices. Open Journal of Discrete Applied Mathematics, 4(1), 1-3.

[7] Rada, J., Rodríguez, J. M., \& Sigarreta, J. M. (2021). General properties on Sombor indices. Discrete Applied Mathematics, 299, 87-97.

[8] Redžepović, I. (2021). Chemical applicability of Sombor indices. Journal of the Serbian Chemical Society, 86, 445-457.

[9] Réti, T., Došlić, T., \& Ali, A. (2021). On the Sombor index of graphs. Contributions to Mathematics, 3, 11-18.

[10] Ghanbari, B. (2021). On the Sombor characteristic polynomial and Sombor energy of a graph. arXiv, arXiv:2108.08552.

[11] Gowtham, K. J., \& Swamy, N. N. (2021). On Sombor energy of graphs. Nanosystems: Physics, Chemistry, Mathematics, $12,411-417$.

[12] Gutman, I. (2021). Spectrum and energy of the Sombor matrix. Milirary Technical Courier, 69, 551-561.

[13] Lin, Z., \& Miao, L. (2021). On the spectral radius, energy and Estrada index of the Sombor matrix of graphs. arXiv, arXiv: 2102.03960.

[14] Wang, Z., Mao, Y., Gutman, I., Wu, J., \& Ma, Q. (2022). Spectral radius and energy of Sombor matrix of graphs. Filomat, In Press.

[15] Furtula, B., \& Gutman, I. (2015). A forgotten topological index. Journal of Mathematical Chemistry, 53, 1184-1190.

[16] Kulli, V. R. (2020). Graph indices, in: Pal, M., Samanta, S., \& Pal A. (Eds.). Handbook of Research of Advanced Applications of Graph Theory in Modern Society. Hershey: Global, pp. 66-91.

[17] Vukičević, D., \& Gašperov, M. (2010). Bond additive modeling 1. Adriatic indices. Croatica Chemica Acta, 83, 243-260.

[18] Cvetković, D., Rowlinson, P., Simić, S. K. (2010). An Introduction to the Theory of Graph Spectra. Cambridge: Cambridge University Press.

[19] Li, X., Shi, Y., \& Gutman, I. (2012). Graph Energy. New York: Springer. 
[20] Li, X., \& Wang, Z. (2021). Trees with extremal spectral radius of weighted adjacency matrices among trees weighted by degree-based indices. Linear Algebra and Its Applications, 620, 61-75.

[21] Das, K. C., Gutman, I., Milovanović, I., Milovanović, E., \& Furtula, B. (2018). Degree-based energies of graphs. Linear Algebra and Its Applications, 554, 185-204.

[22] Shao, Y., Gao, Y., Gao, W., \& Zhao, X. (2021). Degree-based energies of trees. Linear Algebra and Its Applications, 621, (2021) 18-28.

[23] Sachs, H. (1964). Beziehungen zwischen den in einem Graphen enthaltenen Kreisen und seinem charakteristischen Polynom. Publicationes Mathematica (Debrecen), 11, 119-134.

[24] Mitrinović, D. S., \& Vasić, P. M. (1970). Analytic Inequalities. Berlin: Springer.

[25] Ozeki, N. (1968). On the estimation of inequalities by maximum and minimum values. Journal of the College of Arts and Science Chiba University, 5, 199-203.

[26] Godsil, C. D., \& Gutman, I. (1981). On the theory of the matching polynomial. Journal of Graph Theory, 5, $137-144$.

[27] Godsil, C. D., \& Royle, G. (2001). Algebraic Graph Theory. New York: Springer.

[28] Gutman, I. (1977). Acyclic systems with extremal Hückel $\pi$-electron energy. Theoretica Chimica Acta, 45, 79-87.

[29] Mateljević, M., Božin, V., \& Gutman, I. (2010). Energy of a polynomial and the Coulson integral formula. Journal of Mathematical Chemistry, 48, 1062-1068. 